\title{
The femoral insertions of the anteromedial and posterolateral bundles of the anterior cruciate ligament: a radiographic evaluation
}

\author{
Hanno Steckel $\cdot$ Volker Musahl $\cdot$ Freddie H. Fu
}

Received: 18 March 2009/Accepted: 11 June 2009/Published online: 30 June 2009

(C) Springer-Verlag 2009

\begin{abstract}
The aim of this radiographic study was to visualize the femoral insertion sites of the anteromedial (AM) and posterolateral (PL) bundle of the anterior cruciate ligament (ACL) on lateral radiographs in different angles of knee flexion to gain better understanding for arthroscopic femoral tunnel placement in ACL double bundle reconstruction. Four fresh cadaveric knees with an intact ACL were dissected to isolate the AM and PL bundle of the ACL. We obtained lateral radiographs of each knee over the range of $0^{\circ}-90^{\circ}$ flexion in $30^{\circ}$ increments after painting the bundles with a radiopaque tantalum powder. The center of the radiographically marked femoral insertion was defined for each bundle on the lateral roentgenogram. We analyzed the relationship of knee flexion and the projection of the relative position of the femoral insertion sites of both bundles of the ACL on the lateral roentgenogram. The centre of the PL bundle visualized more anterior and distal than the centre of the AM bundle with the knee held in $90^{\circ}$ flexion. The centers of the AM and PL bundle were horizontally aligned when the knee was flexed over $90^{\circ}$. The resulting images allow a radiographic description of the femoral insertion sites of both bundles in different angles of knee flexion. It is essential to be aware of the degree of knee flexion when drilling the femoral tunnels.
\end{abstract}

H. Steckel · V. Musahl · F. H. Fu

Department of Orthopaedic Surgery, University of Pittsburgh,

3471 Fifth Avenue, Suite 1011, Pittsburgh, PA 15213, USA

H. Steckel $(\bowtie)$

Department of Orthopaedic Surgery, University of Göttingen, Robert-Koch-Straße 40, 37073 Göttingen, Germany

e-mail: hanno.steckel@gmail.com
Keywords Anterior cruciate ligament . Anteromedial bundle . Posterolateral bundle . Anatomy $\cdot$ Radiographic study

\section{Introduction}

Anterior cruciate ligament (ACL) tear is a common injury in orthopaedic sports medicine [6]. Current techniques in ACL surgery have been associated with satisfactory longterm results in the majority of patients; however, there remains a subset, up to $30 \%$ of patients with unsatisfactory outcomes. Specifically, patients report difficulties relating to rotational instability and return to previous level of activity $[2,5,11]$.

Techniques in ACL reconstruction have progressed over time. The double bundle technique, which aims at restoring the original anatomy of the ACL by reconstructing the anteromedial (AM) and posterolateral (PL) bundle of the $\mathrm{ACL}$, is gaining popularity $[8,13,17]$. The double bundle structure is a commonly accepted model in describing and understanding ACL anatomy [7, 10]. Evaluating the double bundle structure and reconstruction of the ACL, one should consider anatomic aspects to provide guidelines for an anatomical reconstruction. One important point in ACL reconstruction is the placement of the femoral tunnels. The surgeon has to be aware of the location of both femoral insertion sites when arthroscopy is performed.

The aim of this study is to visualize the radiographic localization of the femoral insertion sites of each bundle with different angles of knee flexion on a lateral roentgenogram to get a better understanding of the double bundle structure in knee kinematics, because the visualization of the femoral attachment sites of both bundles changes with different degrees of knee flexion and does not 
seem to get enough consideration. We hypothesize that the centers of the AM and PL bundle are horizontally aligned when the knee is flexed beyond $90^{\circ}$.

\section{Materials and methods}

Four fresh-frozen cadaver knees ( 4 female) aged from 46 to 57 years with an average age of 51.5 years were used in this study. The specimens obtained had approximately $200 \mathrm{~mm}$ of femur and tibia. There were two left and two right knees. Skin, muscles, patella, patella tendon and the joint capsule were removed; however, the passive stabilizing structures of the joint, including the ACL, medial and lateral meniscus, and the collateral ligaments were left intact. A preparation of the ACL was completed to identify and separate the AM and PL bundles. The femoral insertion sites and the motion pattern of the AM and PL bundles were evaluated macroscopically. The bundles were painted with a radiopaque tantalum powder (325 mesh, Puratronic $^{\circledR}, 99.97 \%$, metals basis, $\mathrm{Nb} 50 \mathrm{ppm}$, Alfa Aesar ${ }^{\circledR}$ Company, Ward Hill, MA, USA). The femur and the tibia were each secured within custom-made cylinders using epoxy compound (Bondo, Bondo Corporation, Atlanta, GA, USA) and fixed to the testing system. A Robotic/ Universal Force-Moment Sensor (UFS) Testing System consisting of a six-degree of freedom manipulator (PUMA Model 726; Unimate Inc., Danbury, CT), a UFS (Model 4015; JR3 Inc., Woodland, CA), and a computer that records joint kinematics was used throughout the study. The robotic manipulator was used to accurately determine the position of the femur with respect to the tibia. Lateral radiographs were obtained with a standard fluoroscope (Mini-View 6800, GE OEC Medical Systems Inc., Salt Lake City, Utah, USA) of each knee over the range of $0^{\circ}$ $90^{\circ}$ flexion in $30^{\circ}$ increments to visualize the femoral insertion sites of both bundles.

The center of the radiographically marked femoral insertion was defined for each bundle on the lateral roentgenogram. The angle formed between a horizontal line and a line connecting the centre of the femoral insertion of each bundle at $0^{\circ}, 30^{\circ}, 60^{\circ}$ and $90^{\circ}$ of knee flexion was assessed (Fig. 1). For statistical analysis, we reported the ranges and the medians of our measurements.

\section{Results}

In all four knees, we were able to identify an ACL with an AM and PL bundle, which we divided surgically for better distinction. The femoral insertion site of the whole ACL was semilunar at the posterior part of the inner surface of the lateral condyle. The AM bundle originated from the

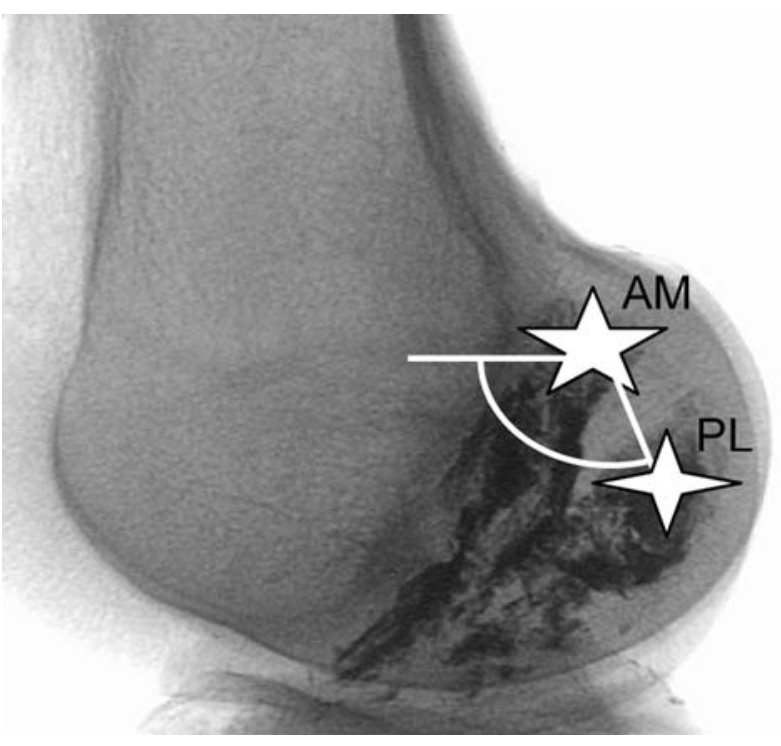

Fig. 1 Schema of angle measurement

anterior-proximal aspect and the PL bundle from the posterior-distal aspect of the femoral attachment with the leg in extension. The motion pattern demonstrated that the AM and PL bundles twist around each other as the knee is flexed toward $90^{\circ}$. The centre of the PL bundle visualized more anterior and distal than the centre of the AM bundle with the knee held in $90^{\circ}$ flexion (Figs. 2, 3, 4, 5). The centers of the AM and PL bundle were horizontally aligned when the knee was flexed beyond $90^{\circ}$. The measurements of the angle between the line connecting the centres of the $\mathrm{AM}$ and PL insertion and a horizontal line demonstrate a decrease of the mean angle with increasing knee flexion toward $90^{\circ}$ (Table 1 ).

\section{Discussion}

The most important finding of the present study was that the visualization of the femoral attachment sites of both

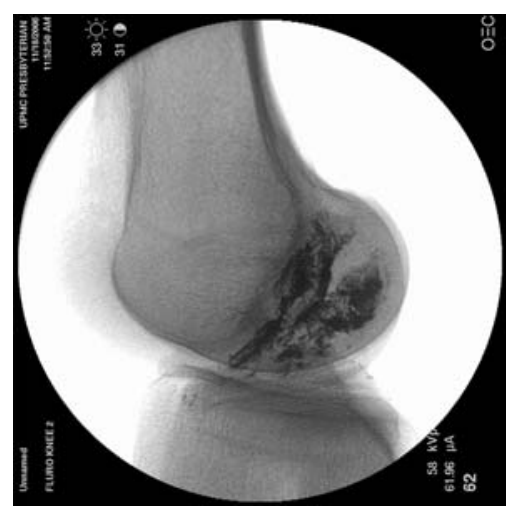

Fig. 2 Lateral radiograph at $0^{\circ}$ of knee flexion 


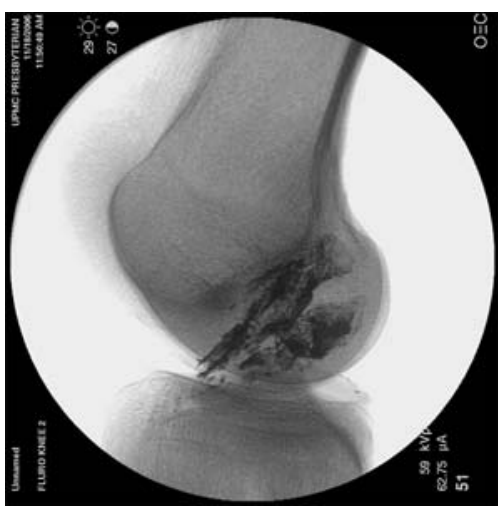

Fig. 3 Lateral radiograph at $30^{\circ}$ of knee flexion

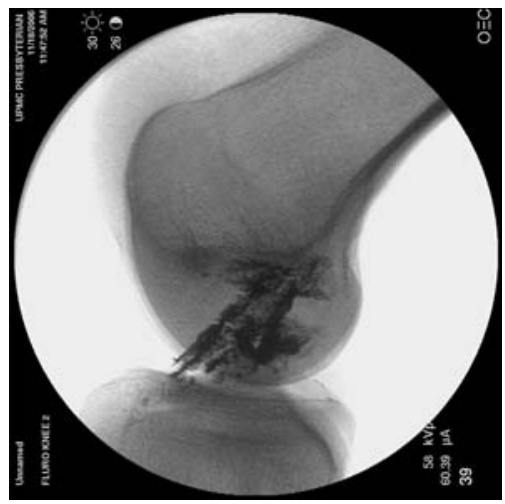

Fig. 4 Lateral radiograph at $60^{\circ}$ of knee flexion

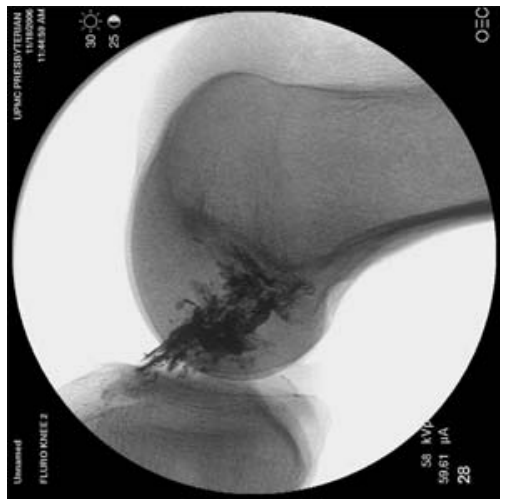

Fig. 5 Lateral radiograph at $90^{\circ}$ of knee flexion

bundles changes with different degrees of knee flexion and that the centers of the AM and PL bundle are horizontally aligned when the knee is flexed beyond $90^{\circ}$. As described in the literature, we found the orientation of the AM and PL bundle to be parallel in the sagittal plane with the knee at full extension, and twisted as the knee was flexed toward $90^{\circ}[7,12,16]$. This observation is a critical point for surgical considerations, because of the flexed knee position used during standard arthroscopy. Consistent with the existing literature, we found the femoral insertion site of
Table 1 Angle measured between a horizontal line and the line connecting the centre of the femoral insertion of AM and PL bundle in the sagittal plane

\begin{tabular}{lllll}
\hline Knee flex. & $0^{\circ}$ & $30^{\circ}$ & $60^{\circ}$ & $90^{\circ}$ \\
Median & 95 & 75 & 51 & 19 \\
Range & $92-111$ & $65-81$ & $45-58$ & $12-31$ \\
\hline
\end{tabular}

the whole ACL to be semilunar at the posterior part of the inner surface of the lateral condyle $[4,10,16]$. The AM and PL bundle originated from the anterior-proximal and posterior-distal aspects of the femoral attachment. The AM bundle inserted anteromedially and the PL bundle posterolaterally, as previously reported [1, 3, 9]. This study reveals the importance of the correlation of knee flexion and femoral insertion site position. It is important to note that the anatomical description is given with the knee in full extension, while surgery is completed with the knee in flexion. With the knee in normal position for arthroscopy, flexion degrees between $70^{\circ}$ and $90^{\circ}$ are reached. In this position, the PL bundle is visualized anterior-distal to the AM bundle $[14,15]$. Bending the knee beyond $90^{\circ}$, a more horizontal position of the bundles' insertion sites is reached. The hypothesis was supported by our results. Although some authors used radiopaque wires or injected radiopaque contrast for imaging the $\mathrm{ACL}$, the use of the radiopaque paint enabled us to visualize the whole ligament. This method was independent of variation in knee size or film-focus distance and reproducible.

\section{Conclusion}

The clinical relevance of this study is that the centers of the AM and PL bundle become horizontally aligned when the knee is flexed beyond $90^{\circ}$. The degree of knee flexion should be taken into account for femoral tunnel placement and for describing tunnel positioning.

\section{References}

1. Amis AA, Dawkins GP (1991) Functional anatomy of the anterior cruciate ligament. Fibre bundle actions related to ligament replacements and injuries. J Bone Joint Surg Br 73:260-267

2. Anderson AF, Snyder RB, Lipscomb AB Jr (2001) Anterior cruciate ligament reconstruction. A prospective randomized study of three surgical methods. Am J Sports Med 29:272-279

3. Arnoczky SP (1983) Anatomy of the anterior cruciate ligament. Clin Orthop Relat Res 172:19-25

4. Bernard M, Hertel P, Hornung H, Cierpinski T (1997) Femoral insertion of the ACL. Radiographic quadrant method. Am J Knee Surg 10:14-22

5. Brandsson S, Kartus J, Larsson J, Eriksson BI, Karlsson J (2000) A comparison of results in middle-aged and young patients after 
anterior cruciate ligament reconstruction. Arthroscopy 16:178182

6. Brown CH, Carson EW (1999) Revision anterior cruciate ligament surgery. Clin Sports Med 18:109-171

7. Duthon VB, Barea C, Abrassart S, Fasel JH, Fritschy D, Menetrey J (2006) Anatomy of the anterior cruciate ligament. Knee Surg Sports Traumatol Arthrosc 14:204-213

8. Fu FH, Shen W, Starman JS, Okeke N, Irrgang JJ (2008) Primary anatomic double-bundle anterior cruciate ligament reconstruction: a preliminary 2-year prospective study. Am J Sports Med 36:1263-1274

9. Girgis FG, Marshall JL, Monajem A (1975) The cruciate ligaments of the knee joint. Anatomical, functional and experimental analysis. Clin Orthop Relat Res 106:216-231

10. Giron F, Cuomo P, Aglietti P, Bull AM, Amis AA (2006) Femoral attachment of the anterior cruciate ligament. Knee Surg Sports Traumatol Arthrosc 14:250-256

11. Goldblatt JP, Fitzsimmons SE, Balk E, Richmond JC (2005) Reconstruction of the anterior cruciate ligament: meta-analysis of patellar tendon versus hamstring tendon autograft. Arthroscopy 21:791-803

12. Hollis JM, Takai S, Adams DJ, Horibe S, Woo SL (1991) The effects of knee motion and external loading on the length of the anterior cruciate ligament (ACL): a kinematic study. J Biomech Eng 113:208-214
13. Kondo E, Yasuda K, Azuma H, Tanabe Y, Yagi T (2008) Prospective clinical comparisons of anatomic double-bundle versus single-bundle anterior cruciate ligament reconstruction procedures in 328 consecutive patients. Am J Sports Med 36:1675-1687

14. Siebold R, Ellert T, Metz S, Metz J (2008) Femoral insertions of the antero-medial and posterolateral bundles of the anterior cruciate ligament: morhometry and arthroscopic orientation models for double bundle bone tunnel placement-a cadaver study. Arthroscopy 24:585-592

15. Steckel H, Starman JS, Baums MH, Klinger HM, Schultz W, Fu FH (2007) Anatomy of the anterior cruciate ligament doublebundle structure: a macro-scopic evaluation. Scan J Med Sci Sports 17:387-392

16. Takahashi M, Doi M, Abe M, Suzuki D, Nagano A (2006) Anatomical study of the femoral and tibial insertions of the anteromedial and posterolateral bundles of human anterior cruciate ligament. Am J Sports Med 34:787-792

17. Yasuda $\mathrm{K}$, Kondo E, Ichiyama $\mathrm{H}$, Kitamura $\mathrm{N}$, Tanabe $\mathrm{Y}$, Tohyama H, Minami A (2004) Anatomic reconstruction of the anteromedial and posterolateral bundles of the anterior cruciate ligament using hamstring tendon grafts. Arthroscopy 20:10151025 